\title{
Leigh disease associated with a novel mitochondrial DNA ND5 mutation
}

\author{
Robert W Taylor ${ }^{*, 1}$, Andrew AM Morris ${ }^{2}$, Michael Hutchinson ${ }^{3}$ and Douglass M Turnbull ${ }^{1}$ \\ ${ }^{1}$ Department of Neurology, The Medical School, University of Newcastle upon Tyne, Framlington Place, Newcastle \\ upon Tyne, NE2 4HH, UK; ${ }^{2}$ Department of Metabolic Medicine, Great Ormond Street Hospital, London, WC1N \\ 3JH, UK; ${ }^{3}$ Department of Neurology, St Vincent's University Hospital, Dublin, Republic of Ireland
}

Leigh disease is a genetically heterogeneous, neurodegenerative disorder of childhood that is caused by defects of either the nuclear or mitochondrial genome. Here, we report the molecular genetic findings in a patient with neuropathological hallmarks of Leigh disease and complex I deficiency. Direct sequencing of the seven mitochondrial DNA (mtDNA)-encoded complex I (ND) genes revealed a novel missense mutation (T12706C) in the mitochondrial ND5 gene. The mutation is predicted to change an invariant amino acid in a highly conserved transmembrane helix of the mature polypeptide and was heteroplasmic in both skeletal muscle and cultured skin fibroblasts. The association of the T12706C ND5 mutation with a specific biochemical defect involving complex I is highly suggestive of a pathogenic role for this mutation. European Journal of Human Genetics (2002) 10, 141 -144. DOI: 10.1038/sj/ejhg/5200773

Keywords: Leigh disease; complex I; mitochondrial DNA; mutation; heteroplasmy

\section{Introduction}

Leigh disease is a neurodegenerative condition with a variable clinical course, though it usually presents during early childhood. It is characterised neuropathologically by symmetrical vasculonecrotic lesions, primarily in the brainstem and basal ganglia, though there may also be abnormalities in the spinal cord, cerebellum, other parts of the brain, optic and peripheral nerves. In most patients, the condition is caused by defects of mitochondrial oxidative phosphorylation, the commonest biochemical abnormalities involving the pyruvate dehydrogenase complex, cytochrome $c$ oxidase (COX) and complex I. ${ }^{1,2}$ Consequently, both nuclear gene defects and mutations of the mitochondrial genome (mtDNA) have been characterised in patients with Leigh disease. ${ }^{1}$

A number of mitochondrial DNA (mtDNA) mutations have recently been reported in association with a specific complex I deficiency in several of these patients. These have included

*Correspondence: Dr RW Taylor, Department of Neurology, The Medical School, Framlington Place, University of Newcastle upon Tyne, Newcastle upon Tyne, NE2 4HH, UK. Tel: +44 191 2228334; Fax: +44 191 2228553; E-mail: r.w.taylor@newcastle.ac.uk

Received 21 September 2001; revised 5 December 2001; accepted 12 December 2001 mutations in the tRNA ${ }^{\text {Leu(UUR) }}$ gene, ${ }^{3}$ and a G14459A transition in the ND6 gene that has previously been characterised in patients with Lebers hereditary optic neuropathy (LHON) and dystonia. ${ }^{4}$ The association of this mutation with Leigh disease therefore raises the possibility that mutations in other mtDNA-encoded structural complex I genes may play a role in the pathogenesis of this disorder. Here, we report the identification of a novel mtDNA mutation in the ND5 gene in a patient with Leigh disease and complex I deficiency.

\section{Materials and methods}

Case report

The detailed clinical, neuropathological and biochemical findings in this patient have been reported previously (Patient 7). ${ }^{2}$ In summary, he presented aged 6 years with left-sided optic atrophy, which subsequently became bilateral. At 20 years of age, he started complaining of leg weakness. Over the next 3 years, he developed ataxia, facial weakness, impaired hearing, ophthalmoplegia and weakness of the muscles of mastication, palate and larynx, necessitating a tracheostomy and gastrostomy. The CSF lactate concentration was $4.0 \mathrm{mmol} / \mathrm{l}$ (normal $<2.2 \mathrm{mmol} / \mathrm{l}$ ). He 
died of peritonitis, aged 24 years. Neuropathology revealed symmetrical foci of neuronal loss, gliosis and microcapillary proliferation in the putamen, periaqueductal gray matter, inferior olives and cerebellar cortex. There was asymmetrical involvement of the medullary tegmentum and central axonal loss in the optic nerves. There is no family history of note, and the patient's mother has declined further investigations.

\section{Muscle histology and biochemistry}

Succinate dehydrogenase (SDH) and cytochrome $c$ oxidase (COX) activities were determined histochemically in cryostat sections $(8 \mu \mathrm{m})$ of frozen muscle. Mitochondrial fractions were isolated from skeletal muscle and cultured skin fibroblasts, and the activities of the individual respiratory chain complexes and the matrix marker citrate synthase were determined spectrophotometrically as previously described. ${ }^{5}$

\section{Mitochondrial DNA analysis}

Total DNA was extracted from both skeletal muscle and fibroblasts using standard procedures. Direct sequencing of all seven mitochondrially-encoded complex I genes was performed as previously described, using M13-tagged PCR primers and BigDye terminator cycle sequencing chemistries (Applied Biosystems, Foster City, CA, USA). ${ }^{6}$

The level of mutated mtDNA was determined by 'last hot cycle' PCR - RFLP analysis using primers corresponding to nt positions 12284-12302 (forward) and nt positions 1281512795 (reverse) to PCR-amplify a 532-bp fragment. Following 30 cycles of amplification, an additional cycle was performed in the presence of $5 \mu \mathrm{Ci}\left[\alpha_{-}{ }^{32} \mathrm{P}\right] \mathrm{dCTP}(3000 \mathrm{Ci} / \mathrm{mmol})$. Labelled products were precipitated and equal amounts (2000 counts) digested with 10 U MnlI (New England Biolabs (UK) Ltd, Hitchin). Restriction fragments were separated through an $6 \%$ nondenaturing polyacrylamide gel, dried onto a support and analysed with ImageQuant software following exposure to a Storm ${ }^{\mathbb{R}}$ PhosphorImager (Molecular Dynamics, Sunnyvale, CA, USA). A single MnlI recognition site in the wild type product generates fragments of 403 and 129 bp. The T12706C transition creates an additional MnlI site, cutting the $403 \mathrm{bp}$ fragment into smaller products of 303 and $100 \mathrm{bp}$, permitting the discrimination of wild-type and mutant mtDNA molecules (see Figure 1).

\section{Results}

Muscle histology revealed minor non-specific changes and the histochemical activities of cytochrome $c$ oxidase (COX) and succinate dehydrogenase were normal. Respiratory chain studies revealed a specific defect in complex I activity in muscle mitochondria (33\% of normal control values). The activities of respiratory chain complexes II, III and IV were all entirely normal (results not shown). The biochemical defect was also expressed in cultured skin fibroblasts, but to a lesser extent ( $49 \%$ of normal control values).
Southern analysis of muscle DNA did not reveal large-scale mtDNA rearrangements, and a subsequent screen for mtDNA mutations known to be associated with Leigh disease (T8993G, T8993C, A8344G) and LHON (G3460A, G11778A, $\mathrm{T} 14484 \mathrm{C})$ proved negative. Direct sequencing of all seven mitochondrially-encoded complex I genes revealed a number of homoplasmic polymorphic variants (MITOMAP database, http://www.gen.emory.edu/mitomap.html) and a single heteroplasmic change from the revised Cambridge reference sequence, a $\mathrm{T}$ to $\mathrm{C}$ transition at position 12706 of the ND5 gene (Figure 1). The ND5 mutation was confirmed as heteroplasmic and shown to be present at levels of $43 \%$ mutant load in skeletal muscle and 30\% mutant load in skin fibroblasts by PCR - RFLP analysis (see Figure 1). Furthermore, the T12706C mutation was not represented in a database of 754 human mitochondrial genomes (MitoKor Mitochondrial SNP Database, personal communication) or detected in 35 patients presenting with mitochondrial encephalomyopathies in whom the causative genetic abnormality was unknown.

\section{Discussion}

We have identified a novel missense T12706C mutation in the mitochondrial ND5 gene in a patient with Leigh disease, and present several lines of evidence to support its aetiologic role. First, it was heteroplasmic and was detectable at higher levels in post-mitotic skeletal muscle than an unaffected tissue (fibroblasts), suggestive of a correlation with the severity of the clinical symptoms. Unfortunately, neuropathological tissue samples were not available for molecular genetic analysis. Second, this mutation was not detected in a large database of 754 human mitochondrial genomes or 35 disease controls and has not been reported previously either in association with optic atrophy or as a common polymorphic variant. Together these data suggest that the T12706C transition does not occur frequently in the general population and provides further evidence of pathogenicity.

Third, the T12706C transition changes an amino acid (phenylalanine-124) in a highly conserved region of the human ND5 protein that is predicted to form one of eighteen transmembrane helices in the protein by structural (Tmpred program) analysis (http://searchlauncher.bcm.tmc.edu/seqsearch/struc-predict.html). Indeed, evolutionary comparison of mitochondrial ND5 sequences indicate that phenylalanine-124 is invariant in all homologous genes (see Table 1). ${ }^{7}$ The mutation predicts an amino acid change to leucine, which also has a hydrophobic side-chain. Whilst the effect of this specific amino acid change on ND5 folding or function cannot be anticipated, a phenylalanine to leucine substitution has been documented previously as pathogenic in a patient with MELAS (mitochondrial myopathy, encephalopathy, lactic acidosis and stroke-like episodes) with a heteroplasmic COX III gene mutation. ${ }^{8}$ 
A
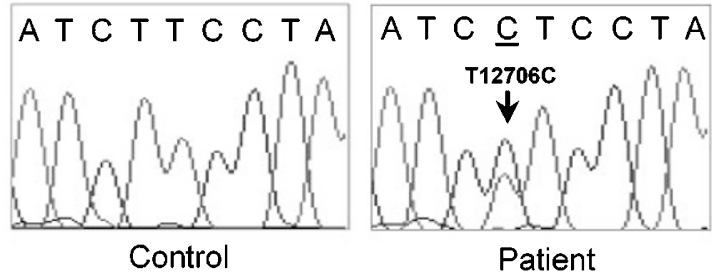

Patient

B
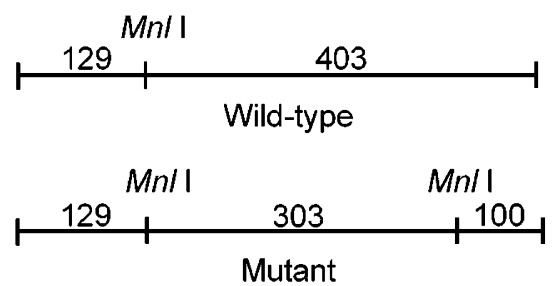

C

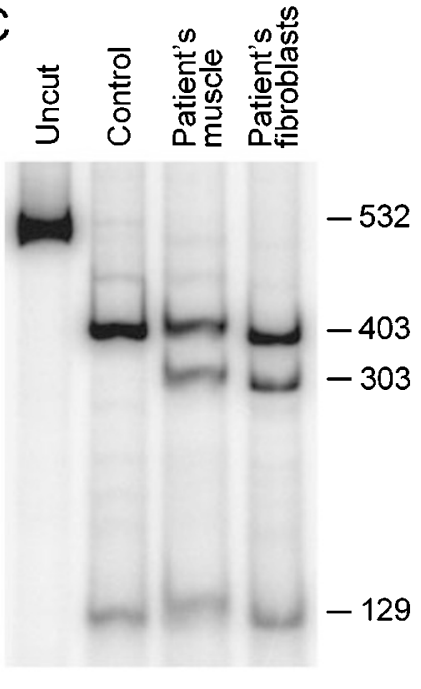

Figure 1 Molecular genetic analysis of the T12706C ND5 mutation. (A) Automated DNA sequence analysis showing wild-type DNA sequence of a region of the ND5 gene from a control and the heteroplasmic T12706C mutation in the equivalent region amplified from the patient. (B) A simple schematic indicating the restriction products that are expected following PCR-RFLP analysis of the ND5 gene region. (C) Phosphorlmage of $6 \%$ nondenaturing polyacrylamide gel showing the $M n l l$ restriction fragments. The level of mutant mtDNA (represented by the $303 \mathrm{bp}$ band) is calculated by quantifying the amount of radioactivity present in the this fragment as a percentage of the combined amount in the 303 and 403 bp (wild-type) fragments after normalising for radiolabeled deoxycytosine content.

Table 1 Multiple sequence alignment of the ND5 gene

\begin{tabular}{|c|c|c|c|c|c|c|c|c|c|c|c|c|c|c|c|}
\hline Patient & 117 & $F F$ & $F \quad K$ & $K Y L$ & $L \quad L$ & 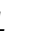 & I & $\underline{L} L$ & L T & $T$ & $T$ & $M$ & $L$ & 1 & \\
\hline Human & & $\mathrm{F} \mathrm{F}$ & $\mathrm{F} K$ & K Y L & L L & & I & F L & L T & & $\mathrm{T}$ & $M$ & $\mathrm{~L}$ & I & L \\
\hline Gorilla & & F F & $\mathrm{F} K$ & $\mathrm{KY} \mathrm{Y}$ & L L & - & I & $\mathrm{F} \mathrm{L}$ & L $\quad T$ & $\mathrm{~T}$ & $\mathrm{~T}$ & $M$ & $\mathrm{~L}$ & 1 & \\
\hline Chimpanzee & & $\mathrm{F} \mathrm{F}$ & $\mathrm{F} \mathrm{K}$ & $\mathrm{K} \mathrm{Y} \mathrm{L}$ & L L & & I & F L & $\mathrm{L}$ & & $\mathrm{T}$ & $M$ & $\mathrm{~L}$ & 1 & \\
\hline Cow & & $\mathrm{F} \mathrm{F}$ & $\mathrm{F} \mathrm{K}$ & $\mathrm{K} Y \mathrm{~L}$ & L L & & $\mathrm{L}$ & $\mathrm{F} \mathrm{L}$ & & & T & $\mathrm{M}$ & $\mathrm{L}$ & I & 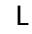 \\
\hline Sheep & & & $\mathrm{F} \mathrm{K}$ & $\mathrm{K} \mathrm{Y} \mathrm{L}$ & $\mathrm{L} \quad \mathrm{L}$ & & $\mathrm{L}$ & $\mathrm{F} \mathrm{L}$ & & & T & $\mathrm{M}$ & & 1 & L \\
\hline Pig & & $\mathrm{FF}$ & & $\mathrm{K} Y \mathrm{~L}$ & L V & & $\mathrm{L}$ & $\mathrm{F} \mathrm{L}$ & L & & T & $\mathrm{M}$ & M & I & t \\
\hline Rat & & $\mathrm{F} \mathrm{I}$ & & K Y L & L $\Lambda$ & M & M & F L & L 1 & $\mathrm{~N}$ & N & $M$ & $\mathrm{~L}$ & 1 & \\
\hline Mouse & & F I & $\mathrm{I} K$ & K Y L & L T & $\Gamma$ & $\mathrm{L}$ & F L & L & & $\mathrm{T}$ & $M$ & $\mathrm{~L}$ & 1 & L \\
\hline Chicken & & $\mathrm{FP}$ & F T & T Y L & L L & & $\mathrm{T}$ & $\mathrm{F} \mathrm{L}$ & L & & A & $\mathrm{M}$ & $\mathrm{L}$ & $\mathrm{T}$ & L \\
\hline Cod & & $\mathrm{FP}$ & & $\mathrm{K} \mathrm{Y} \mathrm{L}$ & L L & & T & $\mathrm{F} \mathrm{L}$ & & V & A & $\mathrm{M}$ & $\mathrm{L}$ & 1 & $\mathrm{~L}$ \\
\hline X. laevis & & $\mathrm{F}$ & $K$ & K Y L & L L & & T & $\mathrm{F}$ & & & A & M & V & 1 & L \\
\hline Earthworm & & $F$ & & L V & V L & - & 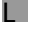 & F & $v$ & & $S$ & $\mathrm{M}$ & $\mathrm{N}$ & M & \\
\hline
\end{tabular}

Evolutionary comparison of the highly conserved region on ND5 sequence (conserved residues are unshaded). The amino acid substitution (phenylalanine to leucine) at postition 124 is shown in bold)

Fourth, and most compelling, the T12706C ND5 mutation clearly associates with a specific biochemical defect involving complex I. The low mutation load of only $43 \%$ in skeletal muscle results in a loss of more than $65 \%$ of control enzyme activity, suggesting that the mutation exhibits a low biochemical threshold for disease expression. This is consistent with other mtDNA-encoded structural gene mutations, ${ }^{9}$ and is in agreement with recent findings in a mouse cell variant carrying a heteroplasmic, nonsense ND5 mutation that demonstrated exquisite control over complex Idependent respiration by this subunit. ${ }^{10}$
This report adds to the growing number of mtDNA mutations involving complex I subunits that have recently been identified in patients with Leigh disease. One of these (G13513A) also resides in the ND5 gene and appears to cause pathology at relatively low levels of heteroplasmy. The G13513A mutation was first described in association with the mitochondrial encephalomyopathy with lactic acidosis and stroke-like episodes (MELAS) phenotype, and has subsequently been reported in patients with $\mathrm{LHON}^{11}$ as well as Leigh disease (DM Kirby, personal communication). The G14459A ND6 LHON/dystonia mutation has also been described at very high levels (>95\%) in three patients with Leigh disease and complex I deficiency. ${ }^{4}$ Interestingly, there was no evidence of optic atrophy in these patients or obvious correlation of the mutant mtDNA load with clinical features.

Our studies in this patient further illustrate the genetic diversity of Leigh disease, and reinforce the importance of maternally inherited mtDNA mutations in the aetiology of this disorder, particularly in those patients who present as adults $^{12}$. Unfortunately, we were not able to study the level of mutation in other maternal relatives as the family declined further investigations and the invitation to attend genetic counselling. Despite the absence of specific treatments for patients with mtDNA disease, we believe that the current momentum to identify and characterise the molecular defects in these patients must be maintained, as this information will have considerable implications for their families both in terms of prognostic advice and genetic counselling. 


\section{Acknowledgments}

RWT and DMT acknowledge the continuing financial support of the Muscular Dystrophy Campaign and the Wellcome Trust.

\section{References}

1 Dahl H-HM: Getting to the nucleus of mitochondrial disorders: identification of respiratory chain enzyme genes causing Leigh Syndrome. Am I Hum Genet 1998; 63: 1594-1597.

2 Morris AAM, Leonard JV, Brown GK et al: Deficiency of respiratory chain complex I is a common cause of Leigh disease. Ann Neurol 1996; 40: 25-30.

3 Kirby DM, Crawford M, Cleary MA, Dahl H-HM, Dennett X, Thorburn DR: Respiratory chain complex I deficiency: an underdiagnosed energy generation disorder. Neurology 1999; 52: $1255-1264$.

4 Kirby DM, Kahler SG, Freckmann M-L, Reddihough D, Thorburn DR: Leigh disease caused by the mitochondrial DNA G14459A mutation in unrelated families. Ann Neurol 2000; 48: 102-104.

5 Taylor RW, Turnbull DM: Laboratory diagnosis of mitochondrial disease; in Applegarth DA, Dimmick JE, Hall JG (eds): Organelle Diseases: Clinical Features, Diagnosis, Pathogenesis and Management. London, Chapman and Hall, 1997, pp 341-350.
6 Taylor RW, Taylor GA, Durham SE, Turnbull DM: The determination of complete human mitochondrial DNA sequences in single cells: implications for the study of somatic mitochondrial DNA point mutations. Nucleic Acids Res 2001; 29: e74.

7 Barker WC, Garavelli JS, Huang H, et al: The Protein Information Resource (PIR). Nucleic Acids Res 2000; 28: 41-44.

8 Manfredi G, Schon EA, Moraes CT et al: A new mutation associated with MELAS is located in a mitochondrial DNA polypeptide-coding gene. Neuromusc Disord 1995; 5: 391-398.

9 Taylor RW, Singh-Kler R, Hayes CM, Smith PEM, Turnbull DM: Progressive mitochondrial disease due to a novel missense mutation in the mitochondrial DNA ND3 gene. Ann Neurol 2001; 50: 104-107.

10 Bai Y, Shakeley RM, Attardi G: Tight control of respiration by NADH dehydrogenase ND5 subunit gene expression in mouse mitochondria. Mol Cell Biol 2000; 20: 805 -815.

11 Pulkes T, Eunson L, Patterson V et al: The mitochondrial DNA G13513A transition in ND5 is associated with a LHON/MELAS overlap syndrome and may be a frequent cause of MELAS. Ann Neurol 1999; 46: 916-919.

12 Chalmers RM, Lamont PJ, Nelson I et al: A mitochondrial DNA tRNA(Val) point mutation associated with adult-onset Leigh syndrome. Neurology 1997; 49: 589-592. 